\title{
Third Harmonic Generation in Hexagonal Boron Nitride Flakes
}

\author{
Anna Popkova ${ }^{1}$, Ilya Antropov', Vladimir Bessonov', Sejeong Kim², Johannes E. Fröch², \\ Igor Aharonovich ${ }^{2}$, Alexander S. Solntsev ${ }^{2}$, and Andrey Fedyanin ${ }^{1}$ \\ ${ }^{I}$ Faculty of Physics, Lomonosov Moscow State University, Moscow 119991, Russia \\ ${ }^{2}$ School of Mathematical and Physical Sciences, University of Technology Sydney, Ultimo, New South Wales, 2007, Australia \\ Authore-mail address: popkova@nanolab.phys.msu.ru
}

\begin{abstract}
The third harmonic generation (THG) in hexagonal boron nitride flakes (hBN) is experimentally studied, the nonlinear third-order susceptibility is estimated.

(C) 2019 The Author(s)

\section{Introduction}

Hexagonal boron nitride is a layered material getting increasing attention. The unusual properties of $\mathrm{hBN}$, such as high optical transparency [1], large bandgap [2], high mechanical strength [3], chemical and thermal stability and excellent oxidation resistivity, made $\mathrm{hBN}$ an outstanding candidate for different electronic and optoelectronic devices. Due to the similar crystal structure to graphene and the smooth surface that is comparatively absent of charge traps and dangling bonds [4], $\mathrm{hBN}$ is also actively used as a substrate for graphene-based technologies [5]. The need for detailed characterization of the $\mathrm{hBN}$ optical response for use one in optoelectronic devices has stimulated a steady and broad array of experimental results [6]. However, the hBN third-order nonlinear optical susceptibility $\chi^{(3)}$, which can have significant impact on all high-power applications, has not been studied until now. In this work, we investigate the THG in $\mathrm{hBN}$ flakes, which have been mechanically exfoliated on a fused silica substrate, and measure $\mathrm{hBN} \chi^{(3)}$.
\end{abstract}

\section{Results and discussion}

The hBN flakes, studied in this work, were prepared by a method previously developed for transition metal dichalcogenides (TMDs) [7]. We obtained a high yield of few-layer hBN flakes. The optical image of a typical flake is shown in Fig. 1(a). It is easy to see that the sample has different color areas, which is caused by the interference of light in thin films, due to the flakes inhomogeneous thickness. $T$ the thicknesses of the sample is determined by atomic force microscopy (AFM) measurements [see Figure 1(b)] andvaries from 5 to $150 \mathrm{~nm}$.
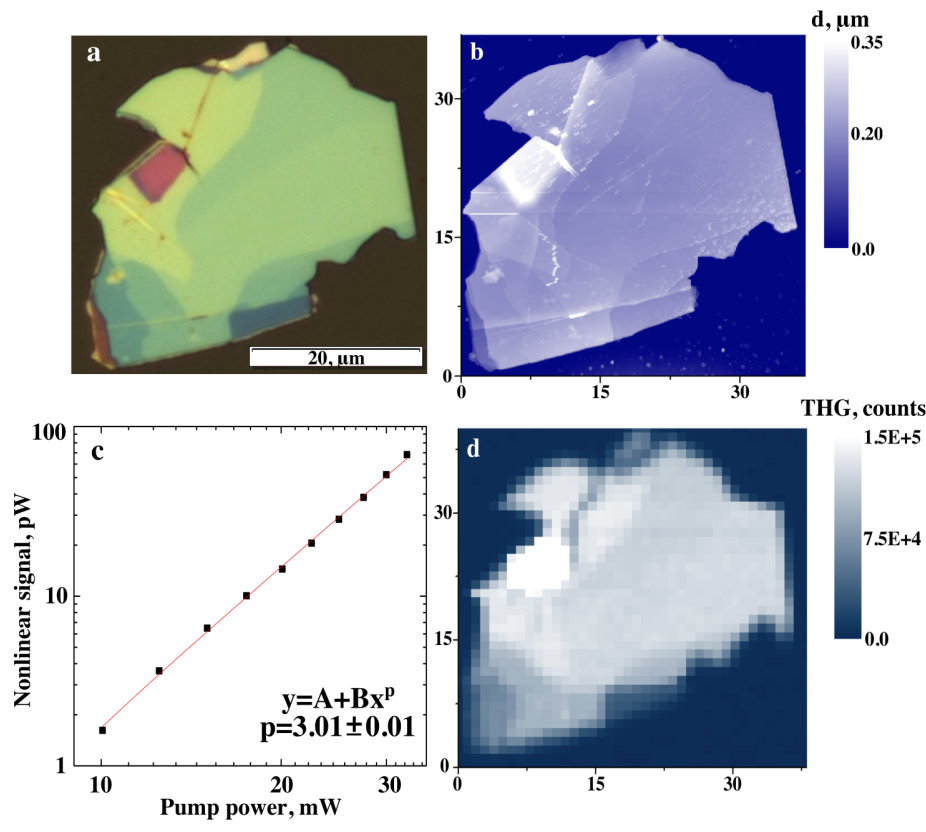

Fig. 1. Images of the hBN flake. (a) Optical image, (b) AFM image. (c) Power-dependent nonlinear optical response of hBN, plotted in double logarithmic scale. (d) THG intensity map. 
The third-order nonlinear susceptibility of an hBN flake was measured using a home-built multiphoton microscope specifically designed for optical harmonics generation. As a light source, a femtosecond Ti:sapphire laser, which can provide laser pulses with a duration of $150 \mathrm{fs}$ and repetition rate of $80 \mathrm{MHz}$ at the $1080 \mathrm{~nm}$ wavelength, was used. The laser beam was focused onto the sample with a $3 \mu \mathrm{m}$ focal spot under normal incidence using the objective lens with an effective NA $=0.25$. Third-harmonic radiation reflected from the sample was collected by the same objective lens and directed by a dichroic mirror to the detection channel for measuring the spectrum of the nonlinear signal or the intensity map [see Fig. 1 (d)].

To confirm the nature of the triple-frequency signal reflected from $\mathrm{hBN}$ flake, we measured its dependence on the pump power [see Fig. 1(c)]. The signal intensity in our case followed the expected cubic dependence on the pump power, which confirmed THG. After that, the polarization of the THG signal was measured. The obtained dependence was approximated with good accuracy by Malus' law, which indicated linear polarization of THG aligned with the polarization of the fundamental beam.

To estimate nonlinear third-order susceptibility of $\mathrm{hBN}$, we measured the THG signal from areas of the flake corresponding to different thicknesses. Unlike second harmonic generation in hBN produced by a single layer due to destructive interference [8], THG is cumulative. In the region of small thicknesses, the dependence of THG signal on flake thickness could be approximated by the quadratic function, which corresponded to the case of no interference between different THG contributions. In thicker regions, a nonmonotonic dependence of THG was observed, which corresponded to the interference between backward THG and frontward THG in reflection from the $\mathrm{hBN} /$ substrate interface. The experimental results were in good agreement with the theoretical THG behavior calculated using the nonlinear transfer matrix method [9]. Based on these results, we estimated the value of the third-order optical susceptibility for $\mathrm{hBN}$ as $\chi^{(3)}=5.1 * 10^{-21} \mathrm{~m} / \mathrm{V}^{2}$.

\section{Conclusion}

To conclude, we have performed the third harmonic generation measurements in hBN flakes and carried out detailed analysis which allowed us to extract the $\mathrm{hBN}$ cubic nonlinear optical susceptibility $\chi^{(3)}=5.1 * 10^{-21} \mathrm{~m} / \mathrm{V}^{2}$.

\section{Acknowledgement}

We acknowledge the funding from the Australian Research Council (DE180100070, DP180100077) and BASIS Foundation (\#19-2-6-28-1).

\section{References}

[1] K. Watanabe, T. Taniguchi and H. Kanda, "Direct-bandgap properties and evidence for ultraviolet lasing of hexagonal boron nitride single crystal." Nature materials 3 (6), 404-409 (2004).

[2] K. Watanabe, T. Taniguchi, T. Niiyama, K. Miya, M. Taniguchi, "Far-ultraviolet plane-emission handheld device based on hexagonal boron nitride." Nature photonics 3 (10), 591 (2009).

[3] C. Zhi, Y. Bando, C. Tang, H. Kuwahara, D. Golberg, "Large-scale fabrication of boron nitride nanosheets and their utilization in polymeric composites with improved thermal and mechanical properties." Advanced Materials, 21 (28), 2889-2893 (2009).

[4] A. V. Krasheninnikov and K. Nordlund, "Ion and electron irradiation-induced effects in nanostructured materials." Journal of applied physics, 107 (7), 3 (2010).

[5] K. K. Kim, et al., "Synthesis of monolayer hexagonal boron nitride on Cu foil using chemical vapor deposition." Nano letters, 12 (1), 161-166 (2012)

[6] J. D. Caldwell, et al., "Photonics with hexagonal boron nitride." Nature Reviews Materials 4 (8), $552-567$ (2019).

[7] S. B. Desai, et al., "Gold-mediated exfoliation of ultralarge optoelectronically-perfect monolayers." Advanced Materials 28 (21), 40534058 (2016).

[8] S. Kim, et al., "Second-harmonic generation in multilayer hexagonal boron nitride flakes," Optics Letters, 44 (23), $5792-5795$ (2019).

[9] D. S. Bethune, 'Optical harmonic generation and mixing in multilayer media: analysis using optical transfer method techniques.' JOSA B 6 (5), 910-916. (1988). 